\title{
Umgang mit urteilsunfähigen Patientinnen und Patienten
}

Die in den letzten Jahren publizierten medizinisch-ethischen Richtlinien der SAMW enthalten unterschiedliche Bestimmungen zum Umgang mit Patientenrechten. Die Zentrale Ethikkommission (ZEK) hat deshalb im Mai 2004 eine Arbeitsgruppe beauftragt, diesbezüglich einen einheitlichen Textbaustein für die zukünftigen Richtlinien zu erarbeiten. Die Arbeitsgruppe «Patientenrechte» ist bei ihrer Vereinheitlichung in erster Linie von den bundesrechtlichen Vorgaben und Entscheidungsspielräumen ausgegangen; sie hat sich weiter an der europäischen Biomedizinkonvention sowie an den allgemeinen Prinzipien der Medizinethik orientiert. Im Herbst 2005 hat die Arbeitsgruppe ihren Vorschlag für «Medizinisch-ethische Grundsätze zum Recht der Patientinnen und Patienten auf Selbstbestimmung» vorgelegt; diese wurden vom Senat der SAMW an seiner Sitzung vom 24. November 2005 verabschiedet. Nachstehend sind die zentralen Inhalte dieser Grundsätze wiedergegeben.

Prof. Dr. iur. Dr. h. c. Kurt Seelmann ${ }^{a}$, Leiter der Arbeitsgruppe «Patientenrechte»

a Ordinarius für Strafrecht und Rechtsphilosophie, Universität Basel
Korrespondenz: lic. iur. Michelle Salathé Schweizerische Akademie der Medizinischen Wissenschaften Petersplatz 13

CH-4051 Basel

m.salathe@samw.ch

\section{Die Bedeutung von Surrogaten}

Die medizinische Behandlung und Betreuung von urteilsfähigen Patienten ist nur rechtmässig beim Vorliegen einer wirksamen Einwilligung. Bei medizinischen Eingriffen an Personen, die selbst zu einer Einwilligung unfähig sind, müssen an die Stelle dieser Einwilligung notgedrungen Surrogate treten: Dazu zählen: eine Entscheidung der Vormundschaftsbehörde, eine schriftlich abgefasste früher gegebene Einwilligung («Patientenverfügung»), eine Einwilligung durch gesetzliche Vertreter oder vom Patienten eingesetzte Vertreter oder eine mutmassliche Einwilligung.

Handelt es sich um Personen, die auch zu keinem früheren Zeitpunkt urteilsfähig waren (originäre Urteilsunfähigkeit), so bleiben von vornherein all diejenigen Surrogate ausser Betracht, die an eine solche frühere Fähigkeit anknüpfen, d. h. ein vom Patienten eingesetzter Vertreter oder eine Patientenverfügung. Es verbleiben dann nur die gesetzliche Vertretung und die (sonstigen) Instrumente des Vormundschaftsrechts. Der gesetzliche Vertreter ist in jedem Fall an das objektive, d.h. medizinisch definierte Wohl des Vertretenen gebunden. In allen anderen Fällen, also wenn der Urteilsunfähigkeit eine Phase der Urteilsfähigkeit vorausgegangen ist (erworbene
Urteilsunfähigkeit), kommen prinzipiell alle genannten Surrogate in Frage. Dann aber stellt sich dringend die Frage nach ihrer Hierarchie im Konfliktfall. Hierbei dürfte das für das Vormundschaftsrecht geltende Subsidiaritätsprinzip dazu führen, dass Entscheidungen der Vormundschaftsbehörde erst dann zum Tragen kommen, wenn andere Surrogate versagen oder ein Interessen- oder Meinungskonflikt vorliegt. Die Praxis zeigt zudem, dass das Erfordernis, bei urteilsunfähigen Patienten primär an die Vormundschaftsbehörde zu gelangen, nicht praktikabel ist, weil relevante medizinische Entscheidungen häufig unter einem gewissen Zeitdruck stehen. Die personellen Ressourcen der Vormundschaftsbehörden gestatten es diesen oft auch nicht, die Funktion der Vertretung vollumfänglich zu übernehmen, so dass die behandelnden Medizinalpersonen die informelle Anweisung erhalten, gemäss dem mutmasslichen Willen des Patienten zu handeln.

\section{Patientenverfügung}

Besonders umstritten ist die Bedeutung der Patientenverfügung. Für die einen gilt sie als verbindliche Willensäusserung des Patienten, für die anderen ist sie (lediglich) ein gewichtiges 
Indiz bei der Ermittlung des mutmasslichen Willens.

Die Arbeitsgruppe hat sich der Auffassung angeschlossen, dass die Patientenverfügung ein gewichtiges Indiz bei der Ermittlung des mutmasslichen Willens ist: Patientenverfügungen sind Vorausverfügungen für eine kaum voraussehbare existentielle Situation und können deshalb weder rechtlich noch ethisch mit einer Jetzt-für-jetzt-Erklärung des urteilsfähigen $\mathrm{Pa}$ tienten verglichen werden. An frühere Einwilligungen dürfen deshalb zumindest keine geringeren Anforderungen gestellt werden als an gegenwärtige. Eine Einwilligung ist grundsätzlich nur bei hinreichender Aufklärung wirksam; eine Patientenverfügung erfüllt diese Voraussetzung jedoch häufig nicht (oft ist die Patientenverfügung ohne jede Aufklärung erfolgt; selbst bei vorgängiger Aufklärung entspricht diese durch Zeitablauf häufig nicht mehr dem gegenwärtigen Stand und sogar wenn sie diesem Stand noch entsprechen sollte, war doch früher das genaue Krankheitsbild mit den konkreten Chancen und Risiken bestimmter Eingriffe nicht vorauszusehen), d.h., der Patient kann sich bei der Abfassung der Patientenverfügung nicht mit einer aktuellen Situation, den Entscheidungsoptionen und -alternativen auseinandersetzen.

\section{Vom Patienten eingesetzter Vertreter}

Die Entscheidung eines vom Patienten eingesetzten Vertreters gleicht in ihrer Bedeutung der Patientenverfügung. Sie ist ein (starkes) Indiz bei der Suche nach dem mutmasslichen gegenwärtigen Willen des Patienten, aber eine ausschliessliche Verbindlichkeit kann auch ihr aus den bei der Patientenverfügung genannten Gründen nicht zukommen. Gegenüber der gesetzlichen Vertretung zeichnet sie sich aber immerhin dadurch aus, dass sie Ausfluss der Selbstbestimmung des Patienten ist. Bei erworbener Urteilsunfähigkeit des Patienten und einer Kollision des (im Zustand der Urteilsfähigkeit) vom Patienten eingesetzten Vertreters mit einem (später eingesetzten) gesetzlichen Vertreter dürfte deshalb die vom Patienten eingesetzte Vertretung grundsätzlich vorgehen (Grundgedanke des Subsidiaritätsprinzips). Der vom Patienten eingesetzte Vertreter ist bei der Einwilligung in Eingriffe ebenso wie der gesetzliche Vertreter an das Wohl des Vertretenen gebunden, kann also auch nur in indizierte Eingriffe einwilligen. Er darf aber, wenn er vom Vertretenen entsprechend beauftragt ist, indizierte und aus medizinischer Sicht notwendige Eingriffe verweigern (in diesem Konfliktfall kann dann das medizinische Personal an die Vormundschaftsbehörde gelangen).

\section{Mutmassliche Einwilligung}

Die mutmassliche Einwilligung gewinnt aus all diesen Gründen zentrale Bedeutung - insbesondere, wenn die Patientenverfügung als (gewichtiges) Indiz im Rahmen einer mutmasslichen Einwilligung betrachtet wird, der gewillkürte Vertreter jedenfalls bei der Zustimmung zu Eingriffen an das Wohl des Patienten gebunden ist und das vormundschaftsrechtliche Instrumentarium nur subsidiär gilt.

Bei der mutmasslichen Einwilligung muss nun aber entschieden werden, wer primär befugt ist, den mutmasslichen Willen festzustellen, auf wessen Mutmassung es also ankommt. Da diese Feststellung immer auch die Vertrautheit mit sehr individuellen Wünschen und zugleich die Kenntnis objektiver «Wohl»-Komponenten erfordert, schlägt die Arbeitsgruppe vor, hier eine Konsensualentscheidung von Angehörigen und behandelnden Medizinalpersonen zu bevorzugen.

Damit wird das Prinzip der Selbstbestimmung nicht etwa eingeschränkt, sondern bei urteilsunfähigen Patienten überhaupt erst - annäherungsweise - durchsetzbar. Die wechselseitige Kontrolle zweier Gruppen, die sich um die Definition des mutmasslichen Willens des Patienten kümmern, kann die Durchsetzung dieses mutmasslichen Willens am besten gewährleisten. 